\title{
ASPECTOS ÉTICOS DEL ENTENDIMIENTO PÚBLICO DE LA BIOTECNOLOGÍA
}

\author{
Bernardo Elias Correa Soares*
}

Resumen: La práctica del control social de la biotecnología demanda salidas bases de naturaleza ética a fin de racionalizar la aplicación de los principios científicos y permitir beneficios para la sociedad en general. Mientras el uso de la tecnología recombinante en productos médicos y farmacéuticos tiene gran aceptación por parte de la sociedad, gracias a la evidente mejora causada por la cura de enfermedades, no está tan claro para el consumidor el valor real de la investigación genética para mejorar la calidad de los alimentos. Es grande la demanda por más información científica para el público a efectos de movilizar la sociedad hacia esclarecimientos en el pensamiento biotecnológico.

Palabras clave: Biotecnología, bioética, transgénico, bioseguridad, genética

\section{ETHICAL POINTS OF VIEW OF BIOTECHNOLOGY’S PUBLIC PERCEPTION}

\begin{abstract}
The practice of biotechnological social control demands ethical bases in order to rationalize the application of scientific principles and for allowing benefits to society. While the use of recombinant technology in pharmaceutical and medical products has great acceptance by society, as consequence of the evident improvement caused by the cure of diseases, the consumer hasn't a clear idea of the real value of genetic research to improve food quality. There is a great demand for more scientific information by the public with the purpose of mobilizing society toward clarifications in the future biotechnological thought.
\end{abstract}

Key words: Biotechnology, bioethics, GMO, biosafety, genetics

\section{ASPECTOS ÉTICOS DO ENTENDIMENTO PÚBLICO DA BIOTECNOLOGIA}

Resumo: A prática do controle social da biotecnologia exige sólidas bases de natureza ética a fim de racionalizar a aplicação dos princípios científicos e permitir benefícios para a sociedade em geral. Enquanto que o uso da tecnologia recombinante nos produtos médicos e farmacêuticos tem grande aceitação por parte da sociedade, graças a evidente melhora causada pela cura de enfermidades, não está tão claro para o consumidor o valor real da pesquisa genética para melhorar a qualidade dos alimentos. A demanda por mais informação científica para o público, é enorme, com a conseqüência de mobilizar a sociedade para esclarecimentos no pensamento biotecnológico.

Palavras chave: Biotecnologia, bioética, transgênico, biossegurança, genética

* Biomédico-microbiólogo y pesquisador sênior del Núcleo de Bioseguridad de la Fundação Oswaldo Cruz (Fiocruz), Rio de Janeiro - Brasil.

Correspondencia: belsoares@globo.com 


\section{Introducción}

Actualmente ya existen cerca de 98,6 millones de acres plantados con variedades agrícolas genéticamente modificadas en todo el mundo, incluyendo soja, maíz, colza (canola), patata (papa) y algodón. El creciente proceso de globalización viene convirtiendo la biotecnología en vehículo de importantes transformaciones económicas y sociales, a la par que crea foros internacionales de debate para discutir las políticas relacionadas con la agricultura y con la industria de los transgénicos. A pesar de la resistencia contra el uso de la biotecnología en el sector alimenticio en los países de la Comunidad Europea, los órganos de evaluación no identificaron ningún riesgo que justifique la prohibición de los productos transgénicos ni fue oficialmente reconocido ningún accidente de grandes proporciones para la salud humana en que esté envuelto el consumo de alimentos genéticamente modificados. Como contraposición, en Estados Unidos, desde 1995 los consumidores norteamericanos no presentan restricciones al comercio de productos transgénicos(1).

En su calidad de mayor exportador de cultivos transgénicos del mundo, Estados Unidos posee reglamentos específicos que contemplan transacciones comerciales involucrando tecnología genética; no obstante, su modelo regulador difiere de la estructura legal adoptada por la Comunidad Europea con relación a los productos de la biotecnología. El gobierno norteamericano considera los productos biotecnológicos de forma semejante a los demás en cuanto a la evaluación de seguridad, no existiendo reglamentación específica para controlar el uso de la ingeniería genética, excepto en los casos de liberación ambiental de organismos genéticamente modificados; en ese caso, agencias gubernamentales inherentes a la agricultura, salud y ambiente actúan específicamente en sus campos de alcance en el control regulativo de procesos tecnológicos, evaluando el riesgo con base en las informaciones pertinentes suministradas por el propio órgano solicitante. Por su parte, el sistema europeo de evaluación de riesgo exige requisitos normalizados de la institución solicitante, la que debe ser capaz de cumplir con esas pautas básicas a fin de actuar con seguridad en la experimentación en biotecnología(2).

Desde la moratoria propuesta en la Conferencia de Asilomar en el inicio de la década del 70(3), varios países están adoptando modelos reguladores propios para el control de la biotecnología, variando de acuerdo con la tradición o con la característica jurídica de cada país. En Brasil, la reglamentación de la tecnología recombinante sigue el modelo europeo, fundado en las ya citadas directivas de la Comunidad Europea. Los límites legales de la biotecnología brasileña fueron establecidos por la instalación de una instancia regulativa compuesta de representantes tanto de la comunidad científica como de consumidores y trabajadores -la Comisión Técnica Nacional de Bioseguridad (CTNBio) - y por un aparato legal, encabezado por la Ley 8.974/95, la así llamada Ley de Bioseguridad. La base biotecnológica en funcionamiento en el país fue incrementada por la reciente ampliación de los programas de investigación de biotecnología y por relevantes estrategias políticas, fundamentadas por el trabajo que están siendo desarrollado por la CTNBio(2,4,5). Tales iniciativas muestran cómo la seriedad y la transparencia de las acciones consolida la cooperación entre los científicos y la sociedad en la construcción del proceso de entendimiento público de la biotecnología.

Considerando el poder que puede ser conferido por el conocimiento de las manipulaciones genéticas, la práctica de control social de la biotecnología demanda salidas base de naturaleza ética, a fin de racionalizar la aplicación 
de los principios científicos y beneficiar a la sociedad en general. En el aspecto de la percepción pública, en ningún momento precedente de la historia la distancia entre la capacidad de entendimiento popular y las reales posibilidades de la ciencia fue tan grande. La aplicación de la biotecnología en la agricultura y en la producción de alimentos está causando una intensa reacción de desconfianza pública: las modificaciones genéticas propuestas por la moderna biotecnología están siendo percibidas de forma diferente por la sociedad que, aturdi$\mathrm{da}$, intenta comprender las informaciones presentadas por los medios de difusión sobre esos avances científicos(5).

Hoy los avances biotecnológicos y los productos alterados genéticamente ya forman parte de la vida cotidiana de los consumidores en todo el mundo. Sin embargo, mientras la tecnología recombinante con finalidades médicas y farmacéuticas ya es bastante aceptada, no está tan claro para el consumidor el valor real de la investigación genética para mejorar la calidad de los alimentos(2). Una indagación reciente realizada en Europa(6) mostró que, mientras la mayoría de los europeos considera útiles y benéficas las aplicaciones farmacéuticas de esta tecnología, otras utilizaciones, sobre todo el uso de la transgenia en la industria de alimentos o la introducción de genes humanos en animales para obtener órganos de trasplante, son consideradas por el público como prácticas cuestionables con gran potencial de riesgo.

\section{Bioética y Biotecnología}

Diaferia(7) observa también que, con el tiempo, el hombre fue desarrollando actividades específicas para perfeccionar el conocimiento biotecnológico, desencadenando una necesaria reflexión ética y moral. Las teorías filosóficas surgidas a partir de este pensamiento establecieron un eje común entre la Biose- guridad, la Bioética y el Bioderecho capaz de consolidar el control de los procesos biotecnológicos, considerando el ámbito de las transformaciones sociales, políticas y económicas y los valores reinantes en la sociedad de este siglo.

La práctica del debate sobre tópicos de biotecnología aproxima a la comunidad y a los científicos, estimulando una mayor toma de conciencia acerca de las normas necesarias para implementar un protocolo de bioseguridad a nivel nacional(2). Por otro lado, el énfasis en la ingeniería genética crea la falsa percepción de que los organismos genéticamente modificados son eventualmente más peligrosos que los organismos no modificados. En muchos casos, la divulgación pública de desarrollos tecnológicos evoluciona hacia controversias políticas y éticas, dificultando un análisis científico de la evaluación de riesgos y de bioseguridad de cada caso(8).

Aunque el conocimiento público de la biología está aumentando, el optimismo sobre las contribuciones de la ingeniería genética para mejorar la calidad de vida está disminuyendo. Por tanto, un mayor nivel de conocimiento no conduce necesariamente hacia una mayor aceptación de parte de la sociedad. Resultados de debates públicos sobre biotecnología muestran que el temor de la población no puede aliviarse estableciendo reglamentos específicos que intentan evitar daños, ya que todavía persisten preconceptos y/o ansiedades sobre la cuestión de la legislación y el riesgo(9).

Con relación a los animales y a los productos transgénicos, una de las mayores preocupaciones del médico veterinario y de otros profesionales relacionados con la temática actual de la salud pública, son los patógenos modificados utilizados en ensayos de investigación. Por ejemplo, la evaluación de seguridad de vectores usados en terapia génica debe orientarse por 
normas éticas de experimentación, con modelos animales de transferencia en vivo de genes, utilizándose el modelo animal o celular más adecuado para aquel estudio.

Las mismas recomendaciones de bioseguridad válidas para los microorganismos humanos se aplican también a los patógenos animales. Además de eso, hay una gran polémica sobre la aceptación de los alimentos genéticamente modificados por parte de los consumidores, resultante, la mayoría de las veces, del alto nivel de desinformación pública acerca de los mecanismos de evaluación de riesgos utilizados por las agencias de control(4).

Albuquerque(10) recuerda que, en la actual sociedad globalizada, varios campos de la vida social y política participan de la noción de "riesgo", aunque con diferentes sentidos y preocupaciones de acuerdo con el proceso involucrado. En el caso de la ciencia, los avances tecnológicos aportan relevantes transformaciones culturales que pueden generar la expectativa de nuevos riesgos.
Por lo tanto, la forma como las personas asocian los alimentos a valores culturales está sufriendo cambios significativos y son necesarias mayores aclaraciones a la población con el fin de intervenir activamente en el proceso de incorporación social de la biotecnología. Es necesario también invertir en instituciones de investigación y en programas estratégicos, que puedan capacitar recursos humanos e incorporar nuevos avances científicos, así como el perfeccionamiento del control de productos transgénicos a través de la información públi$\mathrm{ca}(11)$.

La sociedad desempeña un papel relevante en el debate de las cuestiones éticas y en la confianza hacia la ciencia y la investigación (en especial del área biológica) cada vez más cuestionadas por el público, considerando la necesidad de límites claros y una reglamentación responsable para las prácticas biotecnológicas. La transparencia y el esclarecimiento público contribuyen a aumentar la confianza en las resoluciones de dilemas científicos por parte de la comunidad académica y política. 


\section{Referencias}

1. James C. Global Status of Commercialized Transgenic Crops: 2000. ISAAA Briefs No. 21. [Sitio en Internet]. Disponible en http://www.isaaa.org/publications/briefs/Brief_21.htm Acceso en junio de 2003.

2. Oda LM, Soares BEC. Biodiversity Policies and Recommendations to Promote Sustainable Development in Brazil. In: Oda LM, org. Capacity Building Programme on Biosafety: A Guide to Supervisors. Rio de Janeiro: Editora FIOCRUZ; 1998: 270.

3. Norman C. Genetic Manipulation Guidelines. Nature 1976; 262: 2-4.

4. Oda LM, Soares BEC. Genetically modified foods: economic aspects and public acceptance in Brazil. Trends in Biotechnology 2000; 18 (5): 188-190.

5. Oda LM. Biossegurança no Brasil segue padrões científicos internacionais. Biotecnologia, Ciência e Desenvolvimento 2001; 3 (18): 4-8.

6. European Union in the US. New European Directive on Authorization of GMOs. [Sitio en Internet]. Disponible en http://www.eurunion.org/News/press/2002/2002052.htm Acceso en junio de 2003.

7. Diaferia A. A relação da Biossegurança com o Direito. Journal Associação Nacional de Biossegurança 2001; 1(1): 6 .

8. Garrafa V. Bioethical Radiograph of Brazil. Acta Bioethica 2000; 1: 177-181.

9. Bauer M, Durant J, Gaskell G. European Public Concerted Action Group: Europe Ambivalent on Biotechnology. Nature 1997; 387: 845-847.

10. Albuquerque MBM. Biossegurança: Uma Visão da História da Ciência. Biotecnologia, Ciência e Desenvolvimento 2001; 3 (18): 42 - 45.

11. Oda LM, Soares BEC. Biotecnologia no Brasil: Aceitabilidade Pública e desenvolvimento Econômico. Parcerias Estratégicas 2001; 10: 162-173. 